\title{
Uusioersily
}

\section{P300 brain computer interface control after an acquired brain injury.}

Daly, J., Armstrong, E., Thomson, E., Andreas, P., Gernot, M., \& Martin, S. (2015). P300 brain computer interface control after an acquired brain injury. International Journal on Recent and Innovation Trends in Computing and Communication, 3(1), 318-325.

Link to publication record in Ulster University Research Portal

\section{Published in:}

International Journal on Recent and Innovation Trends in Computing and Communication

Publication Status:

Published (in print/issue): 07/02/2015

\section{Document Version}

Publisher's PDF, also known as Version of record

\section{General rights}

Copyright for the publications made accessible via Ulster University's Research Portal is retained by the author(s) and / or other copyright owners and it is a condition of accessing these publications that users recognise and abide by the legal requirements associated with these rights.

\section{Take down policy}

The Research Portal is Ulster University's institutional repository that provides access to Ulster's research outputs. Every effort has been made to ensure that content in the Research Portal does not infringe any person's rights, or applicable UK laws. If you discover content in the Research Portal that you believe breaches copyright or violates any law, please contact pure-support@ulster.ac.uk. 


\title{
P300 Brain Computer Interface Control after an Acquired Brain Injury
}

\author{
Jean Daly, Elaine Armstrong, Eileen \\ Thomson \\ Cedar Foundation \\ Belfast, Northern Ireland \\ \{j.daly; e.armstrong; e.thomson\}@cedar- \\ foundation.org
}

\author{
Andreas Pinegger, Gernot Müller-Putz \\ Institute for Knowledge Discovery \\ Graz University of Technology, \\ BioTechMed-Graz, \\ Graz, Austria \\ \{a.pinegger; gernot.mueller\}@tugraz.at
}

\author{
Suzanne Martin \\ School of Health Science \\ Ulster University \\ Jordanstown, Northern Ireland \\ s.martin@ulster.au.uk
}

\begin{abstract}
Brain-Computer Interfaces (BCI) are systems that can be controlled by the user through harnessing their brain signals. Extensive research has been undertaken within a laboratory setting with healthy users to illustrate the usability of such systems. To bring these systems to users with severe disabilities it is necessary to develop simple, easy to use systems that can be operated by non-experts outside of the lab and are evaluated by real end users preferably through a user centered design approach. This paper presents a user centered evaluation of a P300 BCI operated by non-expert users in a rehabilitation center with a control group of five healthy participants without acquired brain injury (ABI) and five end users with ABI. Each participant aimed to complete the 30-step protocol three separate times and rate his or her satisfaction from 0 to 10 on the Visual Analogue Scale after each session. Participants then rated their satisfaction with the BCI on the extended QUEST 2.0 and a customized usability questionnaire. The results indicated that end-users were able to achieve an average accuracy of 55\% compared to the control group that reported an average of $78 \%$. The findings indicated that participants were satisfied with the BCI but felt frustrated when it did not respond to their commands. This work was phase one of three to move the BCI system into end users homes. Key recommendations for advancing the P300 BCI towards an easy to use, home-based system were identified, including reducing the complexity of the setup, ensuring the system becomes more responsive and increasing the overall functionality.
\end{abstract}

\section{Keywords- Brain Computer Interface; P300; User Centred Design; Acquired Brain Injury}

$* * * * *$

\section{INTRODUCTION}

Damage to the brain caused by illness or trauma is known as an acquired brain injury (ABI). Advances in medicine and healthcare mean that people are not only surviving ABI but living for longer albeit in some cases with significant residual impairments [1]. Recovery after an ABI depends on the location and the extent of the injury ranging from a complete recovery to complete paralysis. For some people, extended motor paralysis is experienced as Locked in Syndrome (LIS), when a person has limited or no movement, sometimes including certain facial muscles making it difficult to speak even though the person is alert and awake. Traumatic brain injuries and brainstem stroke are considered the largest causes of LIS [2]. Additionally, some long-term cognitive impairments can also impact on attention, concentration, memory, executive functioning, fatigue and behavior post injury. As a result of such an injury people can be socially isolated, have restricted independence and in turn have increased dependence on the caregiver and on the healthcare system [3]. Assistive technologies (AT) can offer a solution to augment function, enable participation and reduce environmental barriers for people who have an acquired brain injury or a complex disability. There is significant gap in the market for accessible devices and services that support the needs for the population with limited muscle activity. Brain Computer Interfaces have the potential to offer access to assistive technology for such a population.

Brain-Computer Interfaces (BCI) are hard and software systems that connect brain waves to external devices allowing people without muscle activity to communicate and control their environment. BCI utilize brain waves elicited by stimulation via non-invasive electrodes placed on the skull to control the system. The stimulation is evoked through different paradigms to enable this control. The P300 event-related potentials (ERP) paradigm requires the user to concentrate on a symbol, mentally counting it each time it is illuminated while ignoring all others; steady-state visual evoked Potential (SSVEP) uses the frequency of flickering lights on the visual cortex; and event-related desynchronization/ synchronization (ERD/ ERS) uses a person e.g., imaged movements as experimental strategy (for more details on these paradigms go to [4-6]). In general the paradigms best fit depends on the person using the system, their needs and the environment [4].

The goal for this novel technology is to increase independence, communication, rehabilitation outcomes, environmental control and social inclusion. Numerous applications have been proven to work with various $\mathrm{BCI}$ paradigms such as spelling [7], Internet browsing [8], entertainment [9], environmental control [10], gaming [1112], neuroprostheses [13], wheelchair control [14] and cognitive rehabilitation [15]. It is evident that BCI can now control a number of applications however little evidence of this is present beyond the laboratory and limited evaluations have been undertaken with participants that would benefit from the use of such a system on a daily basis. The current complexity of BCI systems makes it difficult to transition the technology from the laboratory into real users homes. 
In order to move BCI system towards domestic use on a day-to-day bases by people who could benefit from the system it is essential to engage in user centered design (UCD). UCD seeks to bridge the gap between what the technical developers think would be useful to have on the system and what end users would actually like and use on the BCI [16]. Kübler et al [17] found than less that $10 \%$ of published research on BCI involved real end users. Evidence suggests that UCD increases end user acceptance [18], improves the quality of the system [19], reduces device abandonment [20] [21] and aids the development of a useable system [18]. A number of recent studies have incorporated this approach successfully in the evaluation of BCI for longterm home use with end users with Amyotrophic Lateral Sclerosis (ALS) [12] [22]. Issues such as the complexity of the system set up; the speed and reliability of the BCI response rate; and the type of electrodes and design of the cap were found to be considerable barriers for the everyday use of currently tested BCI with end users [12] [22] [23].

People with ABI can have additional needs as a result of both motor and cognitive impairments. Limited research has explored BCI technology with acquired brain injury end-users [24]. It is recently been identified that some people with ALS can also experience cognitive impairments [25]. Although there is no evidence to indicate that there are specific cognitive abilities required to operate a BCI, Riccio et al [26] suggest attention and working memory play a fundamental role in operation BCI. Polich [27] states that memory processing and selective attention have a direct bearing on controlling a P300 matrix. Kübler et al, [28] highlight the importance of concentration when operating such a system in order to ignore any additional environmental noises, to focus on the symbol that you intend to select and to internally count each time it is illuminated. It is also important to learn how to interact with the system effectively and have the motivation to continue to engage with system [29]. Additionally, it is important to consider the impact on the EEG signal and the signal to noise ratio as a result of an ABI [28]. Limited research has explored the impact cognitive impairment could have on controlling the BCI and the challenges of bringing such systems to this population. The purpose of this study is to identify the usability of BCI systems for end users with cognitive impairment as a result of an ABI through user centered design. The baseline data on the functionality of the BCI was established with a control group of users without ABI in advance of an extensive evaluation phase with individuals with $\mathrm{ABI}$.

\section{MethodS AND MATERIALS}

\section{A. Description of Participants and the Recruitment Process}

Eleven people were recruited to evaluate the prototype. Five participants ( 4 female, $M=36.6$ years, $\pm 9.3 ; N=3$ had no prior experience of $\mathrm{BCI}$ ) were included in a control group described in this study as Healthy Users. A further six potential end users who are living with acquired brain injury (ABI) were recruited ( 1 female, $M=38.5$ years, \pm 8.6 ). All end user participants were post rehabilitation and no longer

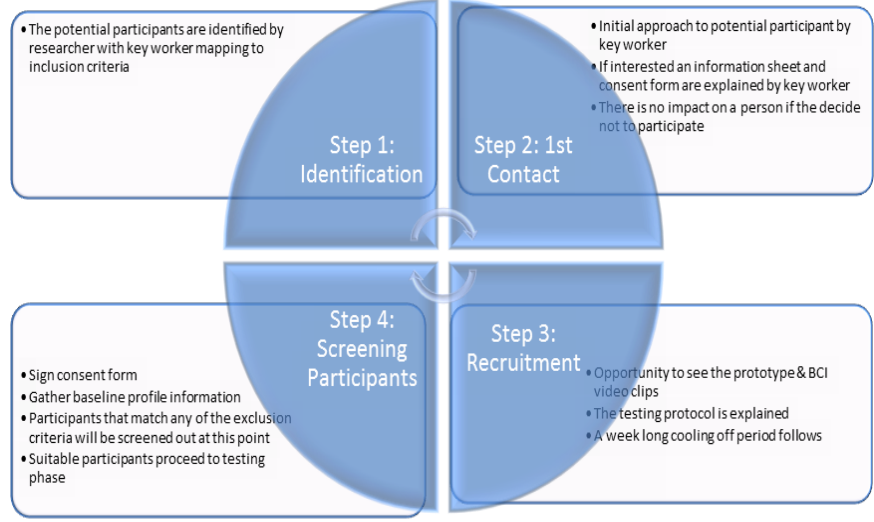

Figure 1. Recruitment of Participants

receiving services from the acute sector. They were medically stable, had no history of epilepsy and had received a diagnosis of moderate to severe brain injury. The degree of cognitive and physical impairment varied although individuals had the cognitive ability to understand the study, the ability to give consent and to learn to interact with the BCI. The mean time post $\mathrm{ABI}$ was 12.17 years $( \pm 6.68)$ ranging from 3 to 24 years and three end users had experience of BCI from a previous project. A detailed requirement process was set out in line with the ethical framework developed for the study that is outlined in Fig. 1. Ethical approval was provided by the University of Ulster Ethics Committee.

\section{B. The BCI Operating System}

The BCI prototype was implemented in Matlab Simulink (MathWorks, USA) and used a P300 based paradigm. One screen displayed the P300 speller matrix, while the other displayed the user interface used for controlling Facebook, Twitter and a desk light (Fig. 2). The distance between user and screen was approximately one meter. EEG was acquired from eight active $\mathrm{Ag} / \mathrm{AgCl}$ electrodes (g.Ladybirds, g.tec, Austria), at the positions Fz, Cz, P3, P4, PO7, POz, Po8 and Oz. The channels were referenced to the right earlobe and a ground electrode was placed at FPz. Signals were amplified by a g.USBamp (g.tec, Austria), sampled at $256 \mathrm{~Hz}$ and band-pass filtered between 0.5 and $30 \mathrm{~Hz}$.

\section{The Evaluation}

The ISO 9241-201 [30] user centered design approach centers on engaging directly with people that could potentially benefit from a product in an iterative process to identify their specific user requirements and to test the systems usability in terms of Effectiveness, Efficiency and Satisfaction. Zickler et al [9] first reported this approach for the evaluation of BCI systems and it was set out further by Kübler et al. [17]. A number of additional studies have also incorporated this approach successfully [12] [22]. The effectiveness of the system is identifying how suitable and usable the system was and this was measured by how accurately the user could make the selection they had intended. The accuracy was calculated by taking the total number of correct selections and dividing this by the total number of selections. 


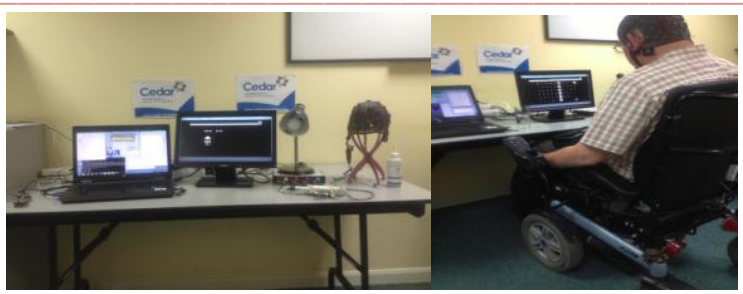

Figure 2. The BCI Prototype and an End User Operating the System

Satisfaction is the identification of the users perception of the system. The visual analogue scale (VAS) questionnaire asked the user to rate their overall satisfaction with the system from zero to ten. The Quebec User Evaluation of Satisfaction with Assistive Technology (QUEST 2.0) is a tool developed to evaluate users satisfaction with assistive technology [31]. Zickler and her team [9] adapted the questionnaire in line with the guidelines set out to maintain the reliability of the measure to include the BCI specific items reliability, speed, learnability, and aesthetic design. Therefore, the ExtendedQUEST 2.0 was adopted to use in this study. Finally, a customized usability questionnaire provided further insight into the users experience of operating the system. The questionnaire included questions on the user preferences with the operating interface, and the positive and negative attributes of the system.

\section{Testing Procedure and Protocol}

The study design set out that each participant would aim to complete a predefined experimental protocol on three different occasions. The experimenter instructed all participants in detail prior to the measurement and checked whether they understood the paradigm before starting the measurement. The set-up phase measured the time from sitting in front of the equipment until commencing the testing protocol. This included placing the cap/electrodes, adding gel, testing the signals, and creating the classifier. For classifier training, users were instructed to consecutively count the number of flashes of five specific letters in a $6 \times 6$ speller matrix. This data was then down-sampled to $64 \mathrm{~Hz}$. Step-wise linear discriminant analysis then automatically determined the most discriminative features from the eight channels and the signal points in the $800 \mathrm{~ms}$ epochs after flash onset and setup the classifier model.

The testing phase required the participant to complete a 30 -step protocol. The researcher guided the participant through the process, which included the selection of fifteen

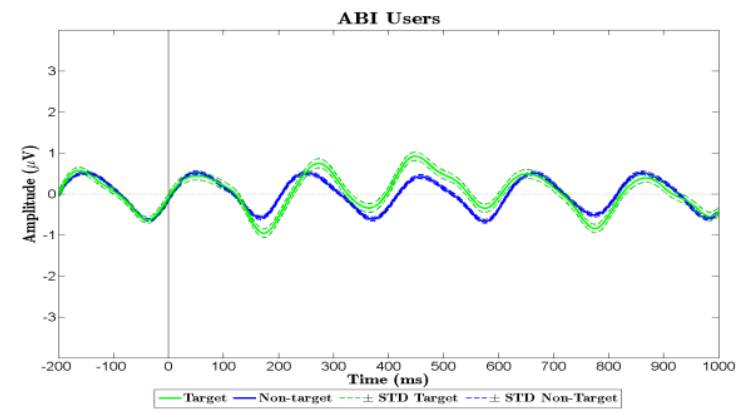

Figure 4. Comparison of the EEG signals of the bipolar derivation Cz-P4 of the ABI (left) and healthy (right) users. The N200 and the P300 amplitude are more pronounced at the healthy users.

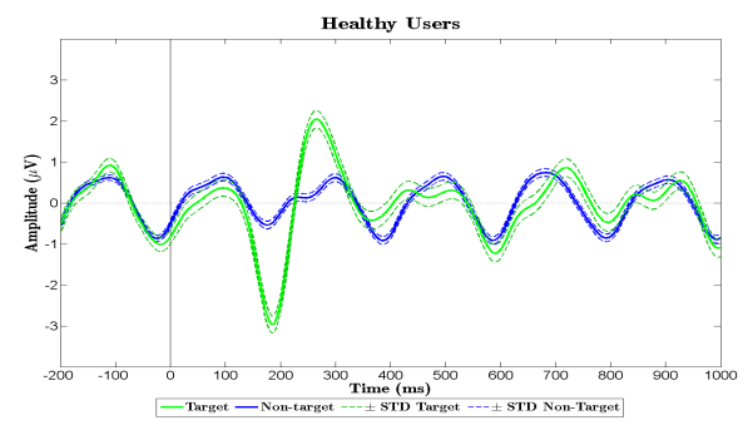

letters (Spelling task) and fifteen selections to navigate the system such as turn on/ off a light and read messages on Facebook or Twitter. Erroneous selections were not corrected. If users were unable to make the correct selection after three attempts the step was abandoned and they were directed to the

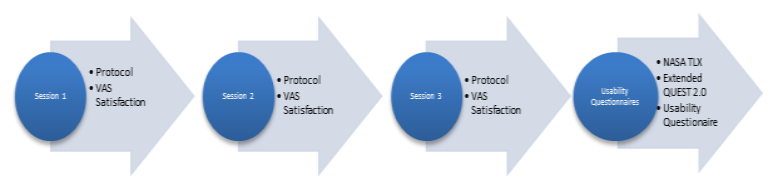

next step in the protocol. The evaluation set out that each participant aimed to complete the protocol in three sessions on three different days, followed by the visual analogue scale (VAS) questionnaire to rate overall satisfaction (Fig. 3). The participant was also invited to give feedback on their session on each occasion. After the final evaluation session participants completed the Extended QUEST 2.0 and the usability questionnaire.

All of the testing was in a rehabilitation center that provides services for people with disabilities in Northern Ireland. The system was placed in a room in this center and there was no effort made to remove noises within the environment so that is was as close to a real life daily experience as possible. A number of noises could be heard at various times such as doors opening or closing, phones, people speaking and moving within the environment.

\section{E. Data Analysis}

Due to the fact that the recorded signals were very noisy the offline signal analysis was performed on bipolar derivations of the electrodes $\mathrm{Cz}$ and $\mathrm{P} 4$. All signals were filtered with a $0.5-15 \mathrm{~Hz}$ band-pass. Only epochs of the training with well-known target and non-target stimuli were used for analysis. The epoch length was $1000 \mathrm{~ms}$. A baseline correction was performed with $200 \mathrm{~ms}$ pre-stimulus data. Both the healthy user and end users signals were checked for statistical significant differences by means of Mann-Whitney U tests. Significant accuracy differences between the healthy control group and the end users as well as between sessions were determined with Kruskal-Wallis tests.
Figure 3. The Evaluation Protocol 

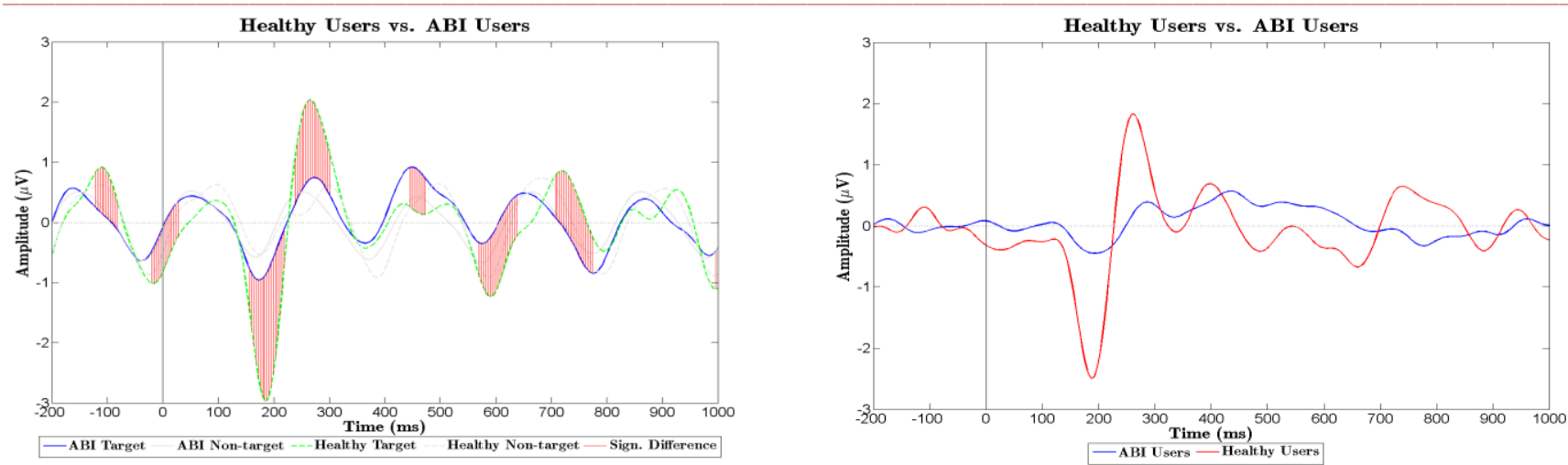

Figure 5. Left: Significant difference $(\mathrm{p}<0.01)$ between the target signals from figure 3 . The plot shows significant amplitude differences at $\mathrm{N} 200$ and P300. Right: Comparison of the differential signals(i.e., target minus non-target) of the ABI and the healthy users.

\section{RESULTS}

\section{A. Set up and Signal Analysis}

All of the set up was undertaken by non-BCI-experts and took 15.5 minutes on average with the healthy control group as oppose to 27.6 minutes for the end users. The most challenging aspect of the set up was to assure good signal quality in all electrodes. Technical and remote support was sought from the technical experts when the non-BCI-experts experienced difficulty in the set up or if the BCI was not responding appropriately.

Differences between the signals of target epochs and nontarget epochs are shown in Fig. 5. The confidence interval of the signals is indicated with dashed lines. The mean peak P300 amplitude of the healthy users was $2.04( \pm 0.22) \mu \mathrm{V}$ and $0.75( \pm 0.11) \mu \mathrm{V}$ for the ABI users. The mean latency of the P300 peak was $265.6 \mathrm{~ms}$ for the healthy and $273.4 \mathrm{~ms}$ for the ABI users. N200 and P300 amplitudes were significantly different between the ABI and the healthy users, see Fig. 5(left). Marked significant differences between the signal of the healthy and the ABI users in Fig. 5(left) except the N200 and P300 areas were originated from phase shifts between these signals. Fig. 5(right) shows the target vs. non-target signal difference for the ABI and healthy users. The difference was much higher for the healthy users.

\section{B. Effectiveness}

All five participants within the healthy control group successfully completed the protocol on three separate occasions. The average time to complete the testing protocol was 15.8 minutes (ranging from 7.1 to minutes). The average selections from the protocol and spelling accuracies of the control group are illustrated in Fig. 6 (below). The average accuracy was $78 \%$ (30 steps) ranging from $65 \%$ to $91 \%$ and the average accuracy to complete the copy spelling was $83 \%$. The overall average accuracy per session and user did not vary significantly $\left(\chi^{2}(2)=0.46, \mathrm{p}=0.80\right)$ between the sessions. Thus, indicating all participants' performance over the three sessions remained relatively stable. Two steps recorded the lowest accuracies when users were trying to make the selections for Facebook (50\%) and Twitter (56\%). A further three steps recorded an average accuracy of below $70 \%(65 \% ; 68 \% ; 68 \%)$ during the protocol. Out of the five least responsive steps four were to navigate the system. The matrix to navigate the system had very few symbols to select from and these findings highlight the importance of matrix size and standardization to optimize performance.

A total of 14 out of 18 attempted protocols were fully completed by the end users. Four protocols were partially completed up to step ten; three of those were stopped as the system was failing to respond and one participant reached the cut off time of two hours using the system. One participant did not complete the evaluation as the system stopped responding to his commands after one complete protocol and two partly completed protocols. The average time taken to complete the protocol was 37.29 minutes (ranging from 12.35 to 64.65 minutes). The evaluation presents an average accuracy of $56 \%$ (range $=41 \%$ to $79 \%$ ) for those completing a full protocol. The four protocols that were partially completed ranged from $36 \%$ to $62 \%$ and this brought the overall average accuracy down to $55 \%$. Additionally, the average accuracy per session (including partially completed sessions) did not vary significantly $\left(\chi^{2}(2)\right.$ $=0.26, \mathrm{p}=0.88$ ) with session one reporting the highest accuracy score of $57 \%$; session two dropped to $51 \%$; and session three was $53 \%$. The average accuracy to complete the copy-spelling task was $61 \%$. Two steps recorded an accuracy score of under $40 \%$ accuracy during the protocol which were the selection for Twitter (36\%) and selecting the 'back' symbol $(37 \%)$ on the final page. Additionally four steps

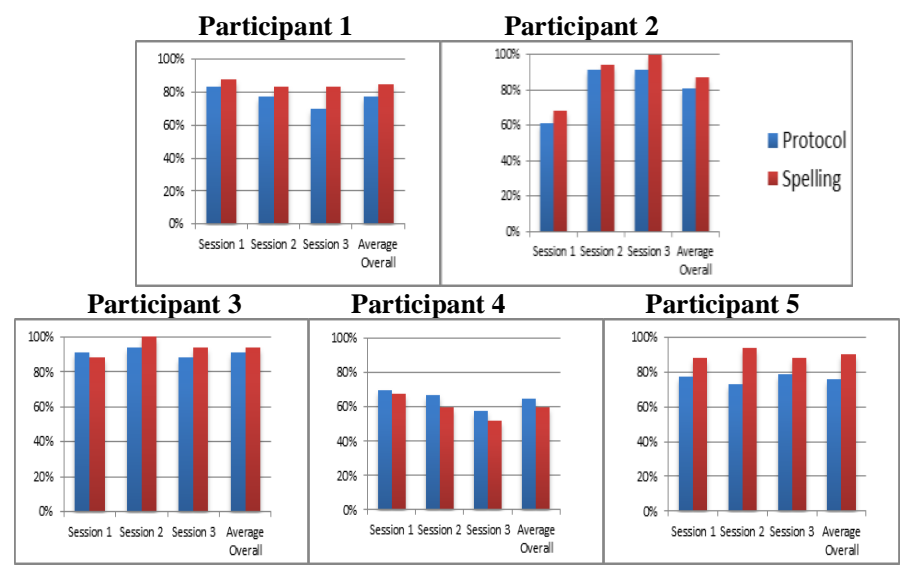

Figure 6. Average accuracies for Healthy Users. 


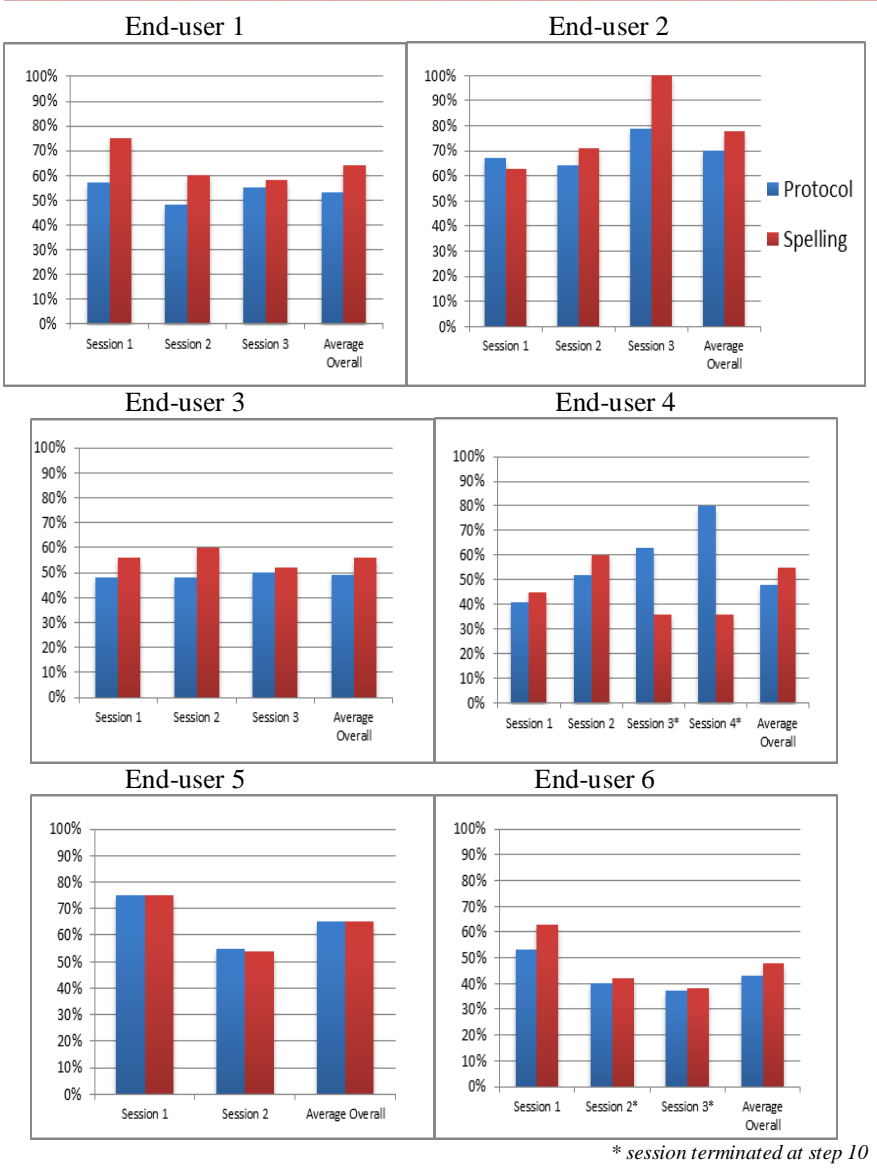

Figure 7. End User Average Accuracy Scores

reported an average accuracy within the $40 \%$ to $50 \%$ range $(41 \% ; 46 \% ; 44 \% ; 45 \%)$. It should be noted that the final four steps of the protocol recorded considerably lower accuracies ranging from $37 \%$ to $45 \%$ especially in comparison to the control group that ranged from $71 \%$ to $83 \%$. This could be attributed to fatigue as a result of the users ABI. Also, each of the steps that reported the lower scores were when the users were navigating the system. This was also found during the health user testing and though to be attributed to the matrix size for the masks during navigation.

\section{User Satisfaction}

Overall device satisfaction reported by the healthy control group on the VAS was 7.6 (range=5-9). The mean QUEST score was 4.23 (4= quite satisfied) and the average score of the added items was 4 . The items rated as most important were ease of use (4), effectiveness (3), speed (3), and reliability (3). The usability questionnaire reported that the control group had a positive experience and felt in charge when using the system. However, only one participant liked the icons on the screen and only two liked the colors. All participants reported that they found it frustrating when the correct selections were not made. Additional comments suggested an ABC versus QWERTY keyboard, Twitter and Facebook selections were difficult to select and the 'afterimaging' made it difficult to make selections. A number of participants also reported that the appearance of the cap and the amount of wires were not appealing.

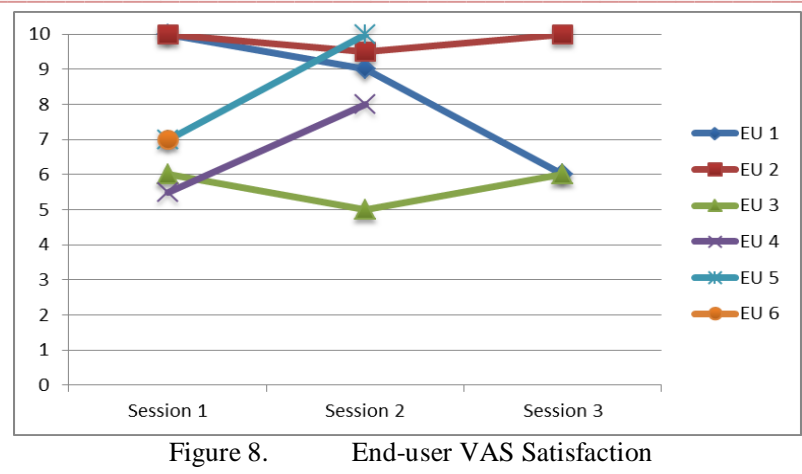

The overall device satisfaction reported on VAS was 7.8 $( \pm 1.9)$ and ranged over the three sessions from 5 to 10 (Fig. 8). The QUEST average score was 3.8 (4= quite satisfied $/ 3=$ more or less satisfied) and the average score of the added items was 3.9. Effectiveness recorded the lowest score on the QUEST (3.4) and speed was highlighted as the dimension end users were least happy with on the Added Items (Table 1). The aspects rated as most important were effectiveness (4), safety (2), comfort (2), ease of use (2), speed (2), and reliability (2).

The usability questionnaire found that end users recognized the potential of the system for the future although they found the system frustrating when it did not respond. Additional comments included difficulty navigating the system using the larger symbols, operating the system required a lot of concentration and it was also tiring. The 'flashing' of the light and the after-imaging some participants experienced was reported as distracting. Ultimately end users were excited about the prospects the system had rather than the systems current functionality.

\section{DISCUSSION}

The present research highlights some positive results as well as a variety of issues that limit the wide spread adoption of BCI technology at the moment. This novel UCD approach engaged with a healthy control group and subsequently worked with end users post ABI. Previous research has indicated that BCI literacy or in other words satisfactory control of the BCI is set at a threshold of 70\% [28] [32]. The control group achieved adequate control of the BCI with an average accuracy score of $78 \%$. The findings indicated that end users with $\mathrm{ABI}$ can operate a BCI, which is promising for future research, however greater control is needed to ensure this technology could become an assistive technology (AT) solution for daily use for people with ABI. The difference between the healthy users and end user group's accuracy score was to be expected and is also evident in previous studies [33] [34] [35]. The importance of the comparison groups is to provide baseline data of the BCI system when non-BCI experts undertake the set up for people with and without a cognitive impairment operate it. Additionally, one end user (EU6) was not able to get adequate control of the BCI over the three sessions and was unable to complete the evaluation. Schreuder et al [23] recommend tailoring the BCI to the needs of the end user by trying a range of paradigms for best fit as opposed to only working with the one paradigm.

A number of recommendations could be considered to increase the systems effectiveness in the future include the use of famous faces as an alternative stimuli to flashing lights on 


\section{TABLE 1. OUTCOME FROM EXTENDED QUEST 2.0}

\begin{tabular}{|c|c|c|c|c|c|c|c|c|}
\hline QUEST & $\begin{array}{l}\text { Healthy } \\
\text { User } \\
\text { Average } \\
\text { Score } \\
\end{array}$ & EU 1 & EU 2 & EU 3 & EU 4 & EU 5 & Average & Comments \\
\hline Dimensions & 4.2 & 3 & 5 & 4 & 5 & 5 & 4.4 & \\
\hline Weight & 4.6 & 3 & 5 & 4 & 5 & 5 & 4.4 & \\
\hline Adjustment & 4.5 & 3 & 4.5 & 3 & 5 & 3.5 & 3.8 & $\begin{array}{l}\text { 'It takes time to } \\
\text { adjust'; 'It crashed } \\
\text { sometimes' }\end{array}$ \\
\hline $\begin{array}{l}\text { Safe and } \\
\text { secure }\end{array}$ & 5 & 3 & 5 & 4 & 5 & 5 & 4.4 & \\
\hline Comfort & 4.8 & 3 & 5 & 3 & 4.7 & 5 & 4.1 & $\begin{array}{c}\text { 'My eyes hurt after } \\
\text { a while'; 'I was a } \\
\text { little tired' }\end{array}$ \\
\hline Easy & 3.8 & 3 & 5 & 2 & 3 & 5 & 3.6 & $\begin{array}{l}\text { 'Sometimes the } \\
\text { system does not } \\
\text { recognise my } \\
\text { command' }\end{array}$ \\
\hline Effective & 3.6 & 3 & 5 & 2 & 2 & 5 & 3.4 & $\begin{array}{l}\text { 'Sometimes the } \\
\text { system does not } \\
\text { recognise my } \\
\text { command' }\end{array}$ \\
\hline $\begin{array}{l}\text { Professional } \\
\text { services }\end{array}$ & 4.8 & 3 & 5 & 3 & 5 & 5 & 4.2 & \\
\hline $\begin{array}{c}\text { QUEST } \\
\text { TOTAL SCORE }\end{array}$ & 4.23 & 2.9 & 4.8 & 3.1 & 4.5 & 4.3 & 3.9 & \\
\hline \multicolumn{9}{|c|}{ Added items } \\
\hline Reliability & 4.4 & 2.5 & 4.8 & 2.8 & 4.5 & 5 & 3.9 & $\begin{array}{l}\text { 'The software was } \\
\text { reliable most of } \\
\text { the time' }\end{array}$ \\
\hline Speed & 4 & 2 & 4 & 2 & 4 & 5 & 3.4 & 'Could be quicker' \\
\hline Learnability & 4.4 & 2 & 3 & 3 & 5 & 5 & 3.6 & \\
\hline $\begin{array}{l}\text { Aesthetic } \\
\text { Design }\end{array}$ & 3.5 & 2 & 5 & 4 & 5 & 5 & 4.2 & \\
\hline $\begin{array}{c}\text { ADDED } \\
\text { ITEMS SCORE }\end{array}$ & 4 & 2.2 & 4.6 & 3.2 & 4.7 & 5 & 3.94 & \\
\hline
\end{tabular}

the P300 matrix [36] [37]; to increase and standardize the size of the matrix in the different applications [6] [38]; and for a simple way to adjust and change the parameters of the stimulus duration to make it easier for the end user to attend to their desired symbol [33]. Additionally, Mulvenna et al [24] suggests personalizing the BCI system for individual needs to increase the lower efficacy rates by users with ABI while Schreuder and his team [23] felt that a range of cognitive profiles need to be taken into account at the design stage of BCI development. A number of biological, psychological and social factors could have impacted on the accuracy score that were not controlled for in this study. For example, the area in the brain where the injury occurred [28]; the participants' motivation level can impact on the EEG signals [29]; and the noise and movement within the social environment can produce strong artifacts that can negatively impact on the overall performance by the user.

There are a range of cognitive skills required to operate the BCI include maintaining attention, concentration, and memory processing whilst ignoring any additional stimuli and environmental noises [26] [27] [28]. The workload required to operate the P300-based BCI was indicated as significant on the usability questionnaires. After an ABI it can be difficult to concentrate for periods of time, maintain attention, process memories as well as decreased levels of stamina. The significant lower N200/P300 EEG response (cf. Fig. 5(left)) supports this statement and indicates that the used paradigm might be challenging for people with ABI. The final four steps of the protocol also highlighted a considerable decrease in control of the BCI by end users. Mental fatigue could be attributed to this reduction in control and it was also indicated as an issue for the end-user group in the usability questionnaire. Thus, the residual cognitive impairment after an
ABI can make it more difficult for the user to operate the BCI but did not prevent the participants in this study from establishing control of the system. Future research should investigate methods of reducing the cognitive workload required to operate BCI systems.

User satisfaction is often strongly linked to the functionality of the BCI [17]. Although the findings indicated that users wanted the response rate of the system increased overall satisfaction with the BCI was recorded on the usability questionnaires as satisfied to very satisfied. The key findings from both groups included frustration when selections were incorrect and difficulty navigating through some aspects of the system. Additional barriers were the complex software and the need for expert support. Equally, both groups recommended changes to the user interface, appearance of the cap/wires, and the control group suggested changing the onscreen keyboard from QWERTY to ABC. Ultimately the users were excited about the prospects the system had rather than the systems current functionality.

A number of issues with the current prototype would prevent the system being used at home on a day-to-day basis. Similar to the previous research, the current response rate by the system, the aesthetic design of the electrode cap and use of gel would be significant barriers for everyday use [12] [22] [23]. Equally, the setup of the system was found to be very complex for non-expert users and the current time necessary to complete the set up will limit its ability to fit into a daily routine [22]. It was particularly difficult to obtain a stable signal from all of the gel-based electrodes. This became even more challenging when setting up the system for the end users with $\mathrm{ABI}$ as can be illustrated by the set up time almost doubling compared to the control group (Table 1). It was difficult for the non-expert user to identify the cause of a system issue and how to resolve this successfully, even with remote support from technical experts. A number of issues could have impacted on the systems response such as insufficient classifier accuracy, misplaced electrode cap, participant fatigue level, a system failure or artifacts in the signal. In order to move the current system forward it would be necessary to support the non-expert user find and resolve issues in order to facilitate the end user to have a positive experience operating the BCI.

\section{CONCLUSION}

The present research aims to develop an easy to use BCI that can be set up by non-expert users, operated by end users with ABI and ultimately bring BCI closer to use in a domestic environment. The work described here is part of an iterative design process to improve the functionality and usability of BCI for users with ABI. The findings have been disseminated to the developers in the hope that future iterations of the prototype will bring BCI closer to the ultimate goal of a commercial available system for home use. Importantly, the findings illustrate that a P300 BCI can be operated by end users with $\mathrm{ABI}$ and therefore has the potential to enhance individual's physical ability, independence and quality of life. 


\section{ACKNOWLEDGMENT}

The research leading to these results has received funding from the European Community's Seventh Framework Programme FP7/2007-2013, BackHome project grant agreement $\mathrm{n}^{\circ} 288566$. We would like to thank all of the project partners for their contribution to this research.

\section{REFERENCES}

[1] World Health Organization, "Neurological Disorders: Public Health Challenges," Geneva: WHO, 2006.

[2] S. Laureys, F. P. Philppe Van Eeckhout, S. Ghorbel, C. Schnakers, F. Perrin, J. Berre et al. "The locked-in syndrome: what is it like to be conscious but paralyzed and voiceless?," Progress in Brain Research, vol. 150, pp.495-611, 2005.

[3] P. Andlin-Sobocki, B. Jonsson, H.U. Wittchen, and J. Olesen, "Cost of disorders of the brain in Europe," European Journal of Neurology,1 vol. 2 (Suppl. 1), pp.1-27, 2005.

[4] C. Guger, G. Krausz, B.Z Allison, and G. Edlinger, "Comparison of dry and gel based electrodes for P300 braincomputer interfaces," Frontiers in Neuroscience, vol. 6, no. 60, pp.1-7, 2012.

[5] P. Durka, R. Kus, J. Zygierewicz, M. Michalska, A. Suda, P. Milanowski, M. Labecki, T. Spustek, and D. Laszuk, "Usercentered design of brain-computer interfaces: OpenBCI.pl and BCI appliance," Bulletin of the Polish Academy of Sciences: Technical Sciences, vol. 60, no. 3, pp.427-431, 2012.

[6] R. Fazel-Rezai, B.Z. Allison, C. Guger, E.W. Sellers, S.C. Kleih, and A. Kübler, "P300 brain computer interface: current challenges and emerging trends," Frontiers in Neuroengineering, vol. 5, no.14, 2012.

[7] A. Kübler, A. Furdea, S. Halder, E.M. Hammer, F. Nijboer, and B. Kotchoubey, "A brain-computer interface controlled auditory event-related potential (p300) spelling system for locked-in patients," Annals of the New York Academy of Sciences, vol. 1157, pp. 90-100, 2009.

[8] I. Käthner, J. Daly, S. Halder, J. Räderscheidt, E. Armstrong, S. Dauwalder, et al. 2014 "A P300 BCI for e-inclusion cognitive rehabilitation and smart home control," In the $6^{\text {th }}$ International BCI Conference , Graz University of Technology, Austria, 2014.

[9] C. Zickler, A. Riccio, F. Leotta, S. Hillian-Tress, S., Halder, E. Holz, P. Staiger-Sälzer, et al. "A brain-computer interface as input channel for a standard assistive technology software," Clinical EEG and Neuroscience, vol. 42, no. 4, pp. 236-244, 2011

[10] J. M Fernández, S. Dauwalder, S. Torrellas, J. Faller, R. Scherer, P. Omedas, P. Verschure et al. "Connecting the disabled to their physical and social world: The BrainAble experience." In TOBI Workshop IV Practical Brain-Computer Interfaces for End-Users: Progress and Challenges, 2013.

[11] A. Finke, A Lenhardt, and H. Ritter, "The MindGame: A P300-based brain-computer interface game," Neural Networks, vol. 22, no. 9, pp. 1329 - 1333, 2009.

[12] E.M. Holz, J. Höhne, P. Staiger-Sälzer, M. Tangermann, and A. Kübler, "Brain-computer interface controlled gaming: evaluation of usability by severely motor restricted end-users," Artificial intelligence in medicine, vol 59, no. 2, pp. 111-120, 2013.

[13] G. Pfurtscheller, G.R. Müller, J. Pfurtscheller, H.J., Gerner, and R. Rupp, "Thought' control of functional electrical stimulation to restore hand grasp in a patient with tetraplegia," Neuroscience Letters, vol. 351, pp. 33-36, 2003.

[14] B. Rebsamen, G. Cuntai, Z. Haihong, W. Chuanchu, T. Cheeleong, M.H. Ang, and E. Burdet, "A brain controlled wheelchair to navigate in familiar environments," IEEE Transactions on Neural Systems and Rehabilitation Engineering, vol. 18, pp. 590-598, 2010.

[15] E. Vargiu, J. Daly, S. Dauwalder, E. Armstrong, S. Martin, and F. Miralles, "Cognitive Rehabilitation through BNCI:
Serious Games in BackHome," $6^{\text {th }}$ International BCl Conference, Graz University of Technology, Austria, 2014.

[16] G. Lightbody, M. Ware, P. Mccullagh, M.D. Mulvenna, E. Thomson, S. Martin, et al. "A user-centred approach for developing brain-computer interfaces," In Proceedings of Pervasive Computing Technologies for Healthcare, Munich, pp. $1-8,2010$

[17] A. Kübler, E. Holz, T. Kaufmann, and C. Zickler, "A user centred approach for bringing BCI controlled applications to end-users" Brain-Computer Interface Systems-Recent Progress and Future Prospects, 2013.

[18] B. Van De Laar, F. Nijboer, H. Gürkök, D.P. Bos, and A Nijholt, "User experience evaluation in BCI: bridge the gap," International Journal of Bioelectromagnetism, vol. 13, no. 3 , pp. 157-158, 2011.

[19] S. Kujala, "User involvement: A review of the benefits and challenges" Behaviour \& Information Technology, vol. 22, no. 1, pp. 1-16, 2003.

[20] A. Shah, and S. Alshawi, "The role of user requirements research in medical device development," In Proceedings of the European and Mediterranean Conference on Information Systems, Abu Dhabi, UAE, 12-13 April, 2010.

[21] J. Abascal, "Human-computer interaction in assistive technology: from 'Patchwork' to 'Universal Design'” In IEEE International Conference on Systems, Man and Cybernetics, Tunisia, October 6-9 2002.

[22] C. Zickler, S. Halder, S.C. Kleih, C. Herbert and A. Kübler, "Brain Painting: Usability testing according to the usercentered design in end users with severe motor paralysis" Artificial Intelligence in Medicine, vol. 59, no. 2, pp. 99-110, 2013.

[23] M. Schreuder, A. Riccio, M. Risetti, S. Dähne, A. Ramsay, J. Williamson, et al. "User-centered design in brain-computer interfaces - A case study". Artificial intelligence in medicine, vol. 59, no. 2, pp. 71-80, 2013.

[24] M. Mulvenna, G. Lightbody, E. Thomson, P. McCullagh, M. Ware, and S. Martin, "Realistic expectations with brain computer interfaces," Journal of Assistive Technologies, vol. 6 , no. 4, pp. $233-244,2012$.

[25] J. Phukan, M. Elamin, P. Bede, N. Jordan, L. Gallagher, S. Byrne, et al. "The syndrome of cognitive impairment in amyotrophic lateral sclerosis: a population-based study," Journal of Neurology, Neurosurgery \& Psychiatry, vol 83, no. 1, pp. 102-108, 2012.

[26] A. Riccio, L. Simione, F.Schettini, A. Pizzimenti, M Inghillen, M.O. Belardinelli, et al. "Attention and P300- based BCI performance in people with amyotrophic lateral sclerosis," Frontiers in Human Neuroscience, vol. 7, pp. 732, 2013.

[27] J. Polich, "Updating P300: an integrative theory of P3a and P3b." Clinical neurophysiology, vol. 118, no. 10, pp. 2128$2148,2007$.

[28] A. Kübler, B. Kotchoubey, J. Kaiser, J.R. Wolpaw, and N Birbaumer. "Brain-computer communication: Unlocking the locked in." Psychological bulletin vol. 127, no. 3, pp. 358-375, 2001.

[29] S.C. Kleih, F. Nijboer, S. Halder, A. Kubler, "Motivation modulates the P300 amplitude during brain-computer interface use," Clinical Neurophysiology, vol. 121, pp. 1023-31, 2010.

[30] ISO-9241-210: Ergonomics of human-system interaction Part 210: Human-centred design for interactive systems. Genf: ISO; 2010. http://www.iso.org/iso/home.html

[31] L. Demers, R. Weiss-Lambrou, and B. Ska, "Quebec user evaluation of satisfaction with assistive technology (QUEST 2.0): An overview and recent progress," Technology and Disability, vol.14, pp. 101-105, 2002.

[32] F. Nijboer, E.W. Sellers, J. Mellinger, M. A. Jordan, T. Matuz, A. Furdea, et al. "A P300-based brain-computer interface for people with amyotrophic lateral sclerosis," Clinical neurophysiology, vol. 119, no. 8, pp. 1909-1916, 2008.

[33] R. Ortner, R. Prueckl, V. Putz, J. Scharinger, M. Bruckner, A. Schnuerer, and C. Guger, "Accuracy of a P300 Speller for Differnet Conditions: A Comparision," In Proceedings of the 
5th International Brain- Computer Interface Confernece, Graz, Austria, September 22 - 24, 2011.

[34] A. Kübler, N. Neumann, B. Wilhelm, T. Hinterberger, and N. Birbaumer, "Predictability of brain-computer communication," International Journal of Psychophysiology, Vol. 18, pp. 121129,2004

[35] F. Piccione, F. Giorgi, P. Tonin, K. Priftis, S. Giove, S. Silvoni, et al. "P300-based brain computer interface: reliability and performance in healthy and paralysed participants," Clinical Neurophysiology, vol. 117, pp. 531-537, 2006.

[36] T. Kaufmann, S.M.Schulz, C. Grunzinger, A. Kubler "Flashing characters with famous faces improves ERP-based brain-computer interface performance," Journal of Neura Engineering, vol. 8: pp. 056016, 2011.

[37] T. Kaufmann, S.M. Schulz, A. Koblitz, G. Renner, C. Wessig and Kubler A. "Face stimuli effectively prevent braincomputer interface inefficiency in patients with neurodegenerative disease," Clinical Neurophysiology, vol. 124, no. 5, pp. 893-900, 2013

[38] E.W. Sellers, D.J. Krusienski, D. J., McFarland, T.M. Vaughan, and Wolpaw, "A P300 event-related potential braincomputer interface (BCI): the effects of matrix size and inter stimulus interval on performance," Biological psychology, vol. 73, no. 3, pp. 242-252, 2006. 